\title{
Research on Edge Detection in License Plate Recognition
}

\author{
Liao $\mathrm{Yu}$ \\ School of Information Engineering \\ Hubei University for Nationalities \\ Enshi 445000,China
}

\begin{abstract}
In traditional recognition system of license plate, the problem is the low recognition rate caused by inaccurate segmentation. This paper proposes a novel adaptive edge detection segmentation algorithm based on first-order differential operator to solve this problem, it can detect the edge of object in vehicle images from complex
\end{abstract}

Keywords-Edge detection, image segmentation, locate license plate, imaging processing

\section{INTRODUCTION}

The recognition system of plate uses advanced image processing and pattern recognition technology to gain the corresponding license plate images from the whole vehicle images, and recognize license automatically. The two main blocks in the recognition system: the segmentation of plate and character ; the recognition systemof plate. The processes of system is shown in Figure.1. If we must achieve real-time recognition on the online traffic, we should provide comprehensive information to the recognition system of license plate [1].

Recently, there were many methods proposesed on edge detection in license plate, such as edge detection based on LMC, Laplace operator, Prewitt operator and Sobel operator, etc[2]. Yet, one of the most fundamental roadblocks has not been overcome. In particular, these methods cannot segment the license plate from the image. As a result, most methods fail to perform well in accurate recogintion.

In this paper, we propose a novel adaptive edge detection algorithm based on first-order differential operator to detect the vehicle images from complex background effectively.

This paper is structured as follows. In Section 2, first we briefly describe the process of image preprocessing and the classic methods in edge detection. Then, a novel algorithm of edge detection is proposed in Section 3. In Section 4, we provide some experimental results in some vehicle images, and we conclude this paper in Section 5.

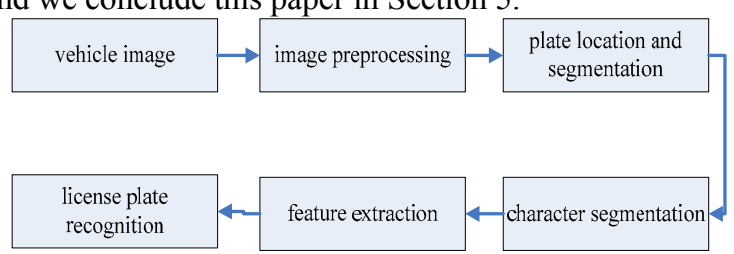

Figure 1. The process of license plate recognition

\section{Edge Detection In License Plate Recognition}

\section{A. Image preprocessing}

The key point of license plate recognition is to locate the plate in image. The vehicle image is low-quality result affected by angle, lighting, weather and other factors. In this process, we must preprocess image to capture the main information of plate. Usually the vehicle image preprocessing includs five steps: grayscale image; image smoothing; image sharpening; binarization and edge detection of license plate. The preprocessing of vehicle image can reduce the complexity of the original image information, locate the license plate simply and fastly, make details clearly. The preprocessing flow chart is shown in Figure.2. In this paper, we mainly research the edge detection on license plate recognition[3-4].

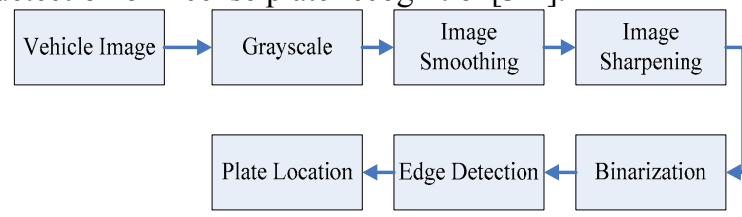

Figure 2. The preprocessing flow chart of vehicle image

\section{B. Edge Detection}

In the recognition, edge detection is vital to image preprocessing. Edge is an important feature in image, we can get some useful informations form it. It, it refers to some pixels with gray value suddenly changed and is composed of a number of straight or curved lines in image. The vchicle image accquismed from various environments, so it may contain diverse informations, such as passer, animal, grass, tree and so on. Although the background of image is complex, the inherent edge character in license plate is obvious. The appearance of plate is a rectangular, the heightwidth ratio of it is a fixed value, and characters arranged horizontally in the rectangular. The size of license plate in china is $440 \mathrm{~cm} \times 140 \mathrm{~cm}$ (length $\times$ width), and the heightwidth ratio is about $3: 1$. There are 7 characters in the license plate, each character is $90 \mathrm{~cm} \times 45 \mathrm{~cm}$, the spacing of edge and character is $10 \mathrm{~cm}$, the spacing of each character is $12 \mathrm{~cm}[5]$. Some gray values changed in the license plate and backguound, characters and plate, these rich characteristices of edge represent a lot of high-frequency componentes in the vechile image. Rich edge informations can be used to get the area of license plate from complex image with two steps: one 
is edge detection for images; the other is segmentation for the plate with inherent edge informations[6].

There are many algorithms on edge detection, such as classical operator, the best operator, multi-scale method and adaptive filtering method. Normally, each method is suitable for different case. We take a brief introduction in traditional Sobel and Prewitt operator in 2-2-1 and 2-2-2. These classic operators have strong ability of denoising, but the cost of calculation is lage[7]. So if you use these operator to process the vehicle images with complex background, they will result in a number of false edges. To solve this problem, we propose a novel adaptive edge detection algorithm based on first-order difference operator to detect the gray edge in vehicle image.

1) Sobel operator

The flow chart of Sobel operator is shown in Figure.3. The mathemetic description in Equation 2-1, 2-2 and 2-3.

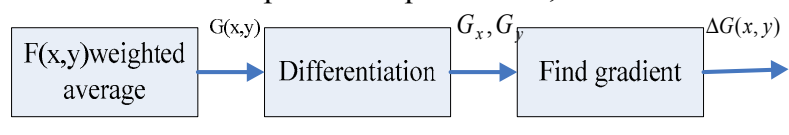

Figure 3. Flow chart of Sobel operator

$$
\begin{aligned}
& \Delta G_{x}=f(x-1, y+1)+2 f(x, y+1)+f(x+1, y+1)-f(x-1, y-1)-2 f(x, y-1)-f(x-1, y-1) \\
& \Delta G_{y}=f(x-1, y-1)+2 f(x-1, y)+f(x-1, y+1)-f(x+1, y-1)-2 f(x+1, y)-f(x+1, y+1) \\
& G[f(x, y)]=\left|\Delta G_{x}\right|+\left|\Delta G_{y}\right|
\end{aligned}
$$

Where $f(x, y)$ is a pixel in the image, $G(x, y)$ is a weighted average of $\mathrm{f}(\mathrm{x}, \mathrm{y}), \mathrm{Gx}$ and Gy is the differentiation of the $G(x, y)$. We solve the gradient of $x$ and $y$ direction respectively to gain the $G[f(x, y)]$.

There are two templates of Sobel operator are shown in Figure.4. The template in the left represents edge of image in the horizontal direction, and in the right represents edge of image in the vertical direction.

\begin{tabular}{|l|l|l|}
\hline-1 & 0 & 1 \\
\hline-2 & 0 & 2 \\
\hline-1 & 0 & 1 \\
\hline
\end{tabular}

\begin{tabular}{|r|r|r|}
\hline \multicolumn{1}{|l|}{1} & \multicolumn{1}{|l|}{-2} & \multicolumn{1}{|l|}{-1} \\
\hline 0 & 0 & 0 \\
\hline 1 & 2 & 1 \\
\hline
\end{tabular}

Figure 4. The template of Sobel operator

\section{2) Canny operator}

Canny operator is used widely in edge detection, it can get good performance in the edge estimation. For the steplike edge, Canny operator is very similar to Gaussian function, so we start this point from Gaussian function.

$$
G(x, y)=\frac{1}{2 \pi \delta^{2}} e^{\frac{x^{2}+y^{2}}{2 \delta^{2}}}
$$

Calcalute the first derivative of $\mathrm{G}(\mathrm{x}, \mathrm{y})$ respect on the the direction $\mathrm{n}$ :

$$
G(x, y)_{n}=\frac{\partial G}{\partial n}=n \cdot \nabla G
$$

$$
\begin{aligned}
& \text { in the type } \\
& n=\left[\begin{array}{l}
\cos \theta \\
\sin \theta
\end{array}\right], \quad \nabla G=\left(\frac{\partial G}{\partial x}, \frac{\partial G}{\partial y}\right)
\end{aligned}
$$

Then processes the point to $\mathrm{f}(\mathrm{x}, \mathrm{y})^{*} \mathrm{G}(\mathrm{x}, \mathrm{y})$, makes the corresponding direction of convoluted maximum is the direction of orthogonal gradient of the edge-trend, namely.

$$
\underline{\partial\left[G_{n} * f(x, y)\right]}=\frac{\partial\left[\cos \theta \frac{\partial G(x, y)}{\partial x} * f(x, y)+\sin \theta \frac{\partial G(x, y)}{\partial x} * f(x, y)\right]}{\partial \theta}=0
$$

This may extract directional derivative of $G_{n} * f(x, y)$, and its the maximum change of directional derivative is shown in Equation 2-8.

$$
n=\frac{\nabla G_{n} * f(x, y)}{\left|\nabla G_{n} * f(x, y)\right|}
$$

It can extract the output on this direction of $n$ simultaneously:

$\left.\left|G_{n} * f(x, y)\right|=\cos \theta(\partial G(x, y) / \partial x) * f(x, y)+\sin A \partial(x, y) / \partial y\right) * f(x, y)|| \mathbf{G}(x, y) * f(x, y) \mid$

From the above Equation 2-9, we can see that it actually obtains two important informations with $\nabla G(x, y) * f(x, y)$. These two informations are the edge intensity and the vetrical direction of edge. From this judgment, the indeed edge is infered by the greatest intensity value on threshold.

\section{OUR Proposed Algorithm}

In view of the problems of above operators, a novel edge detection algorithm based on first-order differential operator is proposed in this paper. In this algorithm, the gray image is binaryed by window with the size of $[-1,0,1]$, $[1,0,-1]$,etc. Of course we must set the threshold as $\mathrm{T}$ during this processing, the value of $T$ varous with the light, shade and different background in the image. In our algorithm, we set $\mathrm{T}$ adaptive in different images[8-9].

$$
g(x, y)= \begin{cases}1, & |f(x-1, y)-f(x+1, y)| \geq T \\ 0, & |f(x-1, y)-f(x+1, y)|<T\end{cases}
$$

And the value of $\mathrm{T}$ is calculated by iteratives in the following steps[10].

Input: the maximum grayscale value in the original image as $Z_{\max }$, the minimum value is $Z_{\min }$;

Step1. have the initial value of threshold is $T_{0}=\left(Z_{\max }+Z_{\text {min }}\right) / 2$

Step2.Uses the initial value of threshold to divide the original gradation into two areas, one is the target as $Z_{o}$, the other is background as $Z_{B}$.

$$
\begin{gathered}
z_{o}=\frac{\sum_{f(i, j)<T_{k}} f(i, j) \times N(i, j)}{\sum_{f(i, j)<T_{k}} N(i, j)} \text { and } \\
z_{B}=\frac{\sum_{f(i, j)>T_{k}} f(i, j) \times N(i, j)}{\sum_{f(i, j)>T_{k}} N(i, j)}
\end{gathered}
$$

Step3. we can obtain a new threshold value like $T_{1}=\left(Z_{O}+Z_{B}\right) / 2$ from the new gradation

Step4. Compare $\mathrm{T}_{0}$ and $T_{1}$, if these two values are equal, then have the best iterative,else go back to the step2.

Output: The final threshold value of T. 


\section{EXPERIMENTS}

In this section, we deal with gray-scale image in experiment. We show the results obtained by canny operator, sobel operator and our proposed algorithm in edge detection.

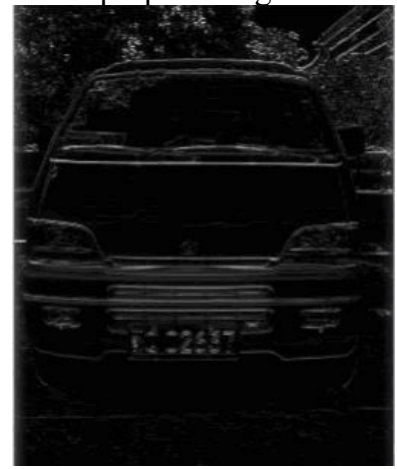

Figure 5. edge detection used by canny operator sobel operator first-order differential operator

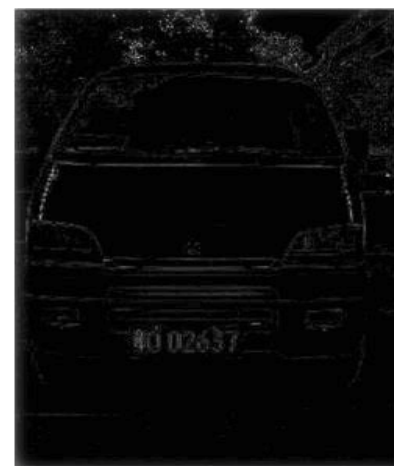

Figure 6. dge detection used by canny operator sobel operator first-order differential operator

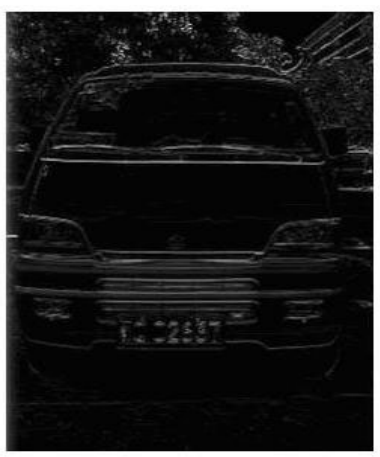

Figure 7. dge detection used by canny operator sobel operator firstorder differential operator

Although the result of canny operator is good, this algorithm wast too much time in computation. The result of sobel operator is very sensitive to noise, many useful edge informations in the original image is diaposed as noise. In Fig.5-7, we can see that the performance of our proposed algorithm is better than other two operator on edge detection. It is denoising effectively and retains details of the edge in original image.
In order to test performance of the proposed algorithm further, we use it in the recongnition of license plate. In the charaacter recognition in plate, the optimized projection and the improved extraction are introduced in this process. The result of this recognition is shown in Fig. 8 - Fig. 10.

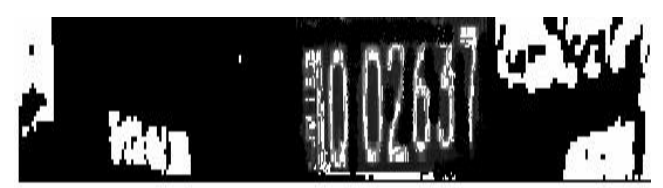

Figure 8 . The sheared result of horizontal projection

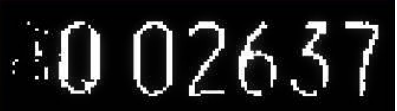

Figure 9. The sheared result of vertical projection

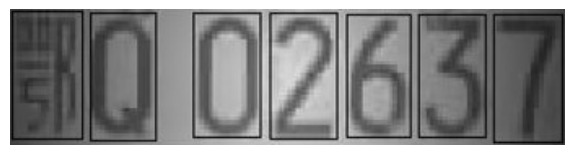

Figure 10. The output of license plate recognition

Finally,we carried above three methods separately on 100 vehicle images to gain the experimental results of license plate recognition in Tab.1:

TABLE I. THE LIST SHOWS RECOGNITION RATE AND WASTING TIME

\begin{tabular}{|c|c|c|}
\hline Algorithm & Recognition rate(\%) & Time(s) \\
\hline $\begin{array}{c}\text { First-order differential } \\
\text { operator }\end{array}$ & 92 & 2 \\
\hline Sobel operator & 78 & 5 \\
\hline Candy operator & 91 & 3 \\
\hline
\end{tabular}

V. CONCLUSION

This paper proposes a novel algorim of edge detection based on the first order differential operator. It can detect the edge of object from complex background effectively. At the same time, an optimized projection and a improved character recognition with 13 feature points also introduced in this paper to solve the character recognition of license plate. Experiments show that our proposed algorithm can achieves state-of-art result.

\section{ACKNOWLEDGMENT}

The work is supported by Science Research Program of Hubei Provincial Department of Education (Q20111907;D20101903), and the Science Research Program of Hubei Provincial Science \& Technology Department under Grant (2010CDB00901).

\section{REFERENCES}

[1] Ostu N.A threshold selection method from gray-level $\begin{array}{lll}\text { histograms[J].IEEE } & \text { Trans } & \text { System,Man, and }\end{array}$ Cybernetics,1979,9(1),PP:62-66. 
[2] Guo Sen,Liu Wei,Wang JingHua.Touched Human Object Segmentation Based on Mean Shift[J].Proceedings of International Symposium on Computer Science and Computational Technology[C].USA:iscsct,2008,PP:29-33.

[3] Roger Y Tsai.An Effic nl and Accurate Camera Calibration Technique for 3-D Machine Vision[J].IEEE Journal of Robotics and Automation,1987,34,PP:323-344.

[4] Juyang Weng,Paul Cohen,Marc Herniou,Camera Calibration with Distoflion Model and Accuracy Evaluation[J].IEEE Trans On Pattern Analysis And Machine Intelligence,1992,14,PP:965-980.

[5] LiGuo, Yu Liao, Daisheng Luo, Honghua Liao. Generic object detection using improved gentleboost classifier.2010 2nd International Conference on Multimedia Intelligence. September, 2010, pp:5291-5296

[6] Mei Yu, Yong Deak Kim. An approach to Korean license plate recognition based on vertical edge matching $[\mathrm{J}] .2000$ IEEE
International Conference on Systems, Man, and Cybernetics, 2000(4),PP:2978-2980.

[7] Chen Liying,Wu Shunhua,Mao Luhong,et al.Design of an analog front end for passive UHF RFID transponderIC[J].Chinese Journal of Semiconductors, 2007,28 (5),PP:686-691.

[8] Kam A.H,Ng T.T, Kingsbury N.G.. Content based image retrieval through object extraction and querying, Proc. IEEE Workshop on Content-based Access of Image andVideo Libraries(CBAIVL),2000,pp:91-95.

[9] ChangSL, ChenLS, ChungYC,etal.Automatic license plate recognition.IEEE Transactions on Intelligent Transportation Systems,2004,5(3),PP:42-53.

[10] M. Nilsson, J. Nordberg, I. Claesson. Face Detection using Local SMQT Features and SplitUp SNoW Classifier, IEEE International Conference on Acoustics, Speech, and Signal Processing(ICASSP),2007,2(4), pp: 589-592. 